\title{
Application of the Front-Fixing Method to Numerical Modeling of Field Diffusion in Nonlinear Systems
}

\author{
Igor O. Golosnoy and Jan K. Sykulski \\ School of Electronics and Computer Science, University of Southampton, Southampton SO17 1BJ, U.K.
}

\begin{abstract}
Application of a finite volume front-fixing method to a thermistor problem with strongly nonlinear material properties is discussed. Advantages and implementation problems of the method are highlighted. Particular attention is paid to conservation properties of the algorithm and accurate solutions close to the moving transition region. The algorithm is compared with other popular numerical techniques. The gains in reduced computational effort and increased accuracy are emphasized.
\end{abstract}

Index Terms-Diffusion processes, moving boundary, numerical analysis, thermistors.

\section{INTRODUCTION}

A THERMISTOR is a circuit device with a very nonlinear dependence of electric conductivity $\sigma$ on temperature $T$ [1]. The changes in $\sigma$ may be rapid; typical $\sigma$ variations of four orders of magnitude with $T$ increasing from about $100{ }^{\circ} \mathrm{C}$ to $200{ }^{\circ} \mathrm{C}$ have been recorded [2] (Fig. 1). The transition region for a given point between temperatures $T_{1}$ and $T_{2}$, which corresponds to coordinates $s_{1}$ and $s_{2}$, is typically small and a coupled treatment of electric and thermal fields is complex. It is quite common to ignore the transition and consider a sharp interface with a step behavior in $\sigma$ [3], [4]. But it was noted [2], [5] that the step function is not the most realistic model for $\sigma$ and that a more complex relationship $\sigma(T)$ should be used for accurate predictions.

This paper deals with modeling the coupled electric current and heat flow in a thermistor with particular attention paid to a moving transient region. The motion is assumed to be a complex function of the solution itself. Many numerical methods developed previously, such as front tracking or remeshing techniques, are often not able to cope effectively with such strong coupling. On the other hand, the front-fixing transformations [6] introduce a coordinate system in which all of the spatial region boundaries are fixed to $s_{1}$ and $s_{2}$. One advantage of discretizing in the transformed space is that the meshes automatically adjust themselves to accommodate the moving interface position. It is therefore possible to impose irregular meshes with fine resolution in regions where large temperature and field gradients are expected, while using larger space steps elsewhere.

The main challenges are the implementation of conservation laws at the moving boundaries and an effective mesh refinement. These issues are addressed in this paper.

\section{Formulation of the Thermistor PROBlem}

\section{A. Geometry of the Device and Material Properties}

Typically, the device is a ceramic disk with a diameter $r_{\text {ext }}$ of about $5 \mathrm{~mm}$ and thickness $\left(2 z_{\text {ext }}\right)$ of about $2 \mathrm{~mm}$ with a wire

Manuscript received October 07, 2008. Current version published February 19, 2009. Corresponding author: I. O. Golosnoy (e-mail: ig@ecs.soton.ac.uk).

Color versions of one or more of the figures in this paper are available online at http://ieeexplore.ieee.org.

Digital Object Identifier 10.1109/TMAG.2009.2012634

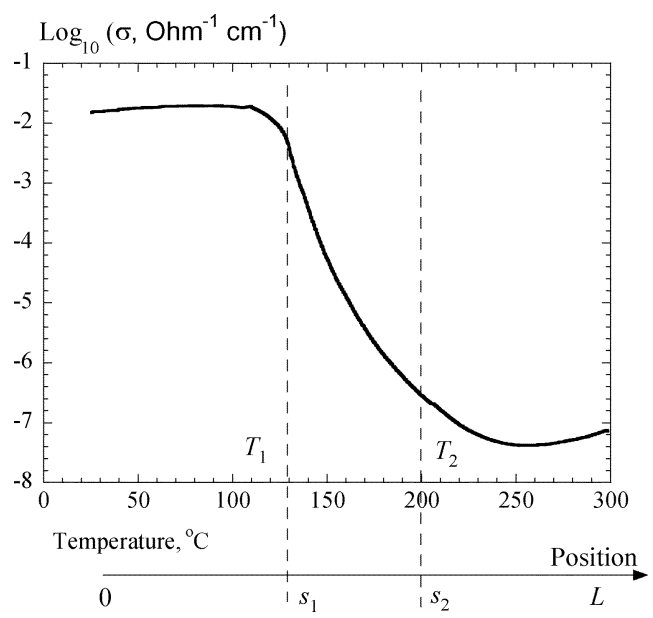

Fig. 1. Typical variation of conductivity with temperature for a thermistor.

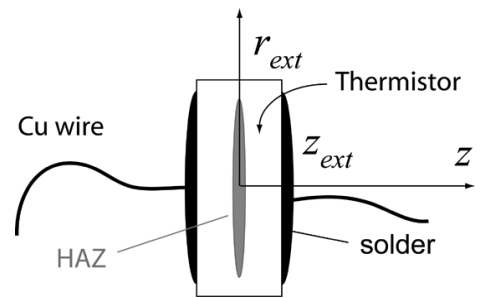

Fig. 2. Geometry of the thermistor device.

soldered to the flat edges [2]. The material properties used for the modeling are identical to those given in [2].

\section{B. Electric Circuit and Governing Equations}

The electric current flow in the system and associated Joule heating are described by Ohm's law

$$
\nabla \mathbf{J}=0, \quad \mathbf{J}=\sigma \nabla \phi, \quad Q_{e l}=\sigma^{-1} \mathbf{J}^{2}=\sigma(\nabla \phi)^{2}
$$

where $\mathbf{J}$ is the current density, $\phi$ is an electric potential, and $Q_{e l}$ is a Joule heat. For simplicity, the electric circuit includes external voltage $V_{0}$ applied to a load $R_{0}$ in series with the thermistor.

When high current passes through the thermistor, Joule heat is released in the middle of the device. The heat affected zone (HAZ) with low conductivity develops inside the thermistor. It 
has initially a complex shape but for $r_{\text {ext }} \gg z_{\text {ext }}$, it almost immediately spreads to the edges, and the transformation front becomes straight and the thermistor problem can be considered by using a 1-D approximation with all parameters depending on the $z$ coordinate only. The current density is linked with the external load and the variable conductivity inside the device

$$
V_{0}=J\left(\pi r_{\mathrm{ext}}^{2} R_{0}+2 \int_{0}^{z_{\mathrm{ext}}} \frac{d z}{\sigma(T(z))}\right) .
$$

It is coupled with the heat transport which is governed by the heat diffusion equation

$$
C \rho \frac{\partial T}{\partial t}=\frac{\partial}{\partial z}\left(\kappa(T) \frac{\partial}{\partial z} T\right)+\sigma^{-1} J^{2}
$$

where $C$ is the specific heat, $\rho$ is the density, and $\kappa$ is the thermal conductivity. Appropriate initial and boundary conditions for (1)-(3) are discussed elsewhere [2]. In the form just shown, (1)-(3) provide an excellent case for testing various numerical techniques to solve coupled diffusion problems.

\section{NumericAl Modeling TeChNIQUeS}

\section{A. Fixed Grid}

Second-order approximations in space and time require semiimplicit finite-difference approximation for (3) [7]. Integrals of (3) around each node $i$ over one time step provide a set of conservative equations in the bulk material [8], which are represented on a fixed grid by (4), where a subscript notation to denote discretizations of space (e.g., $z_{i}$ ) is used and superscripts (e.g., $t^{j}$ ) indicate time

$$
\begin{gathered}
\left(T_{i}^{j+1}-T_{i}^{j}\right)\left(z_{i+0.5}-z_{i-0.5}\right) \\
=\left(t^{j+1}-t^{j}\right)\left(\frac{\kappa_{i+0.5}}{C \rho} \frac{T_{i+1}^{j+0.5}-T_{i}^{j+0.5}}{z_{i+1}-z_{i}}\right. \\
\left.-\frac{\kappa_{i-0.5}}{C \rho} \frac{T_{i}^{j+0.5}-T_{i-1}^{j+0.5}}{z_{i}-z_{i-1}}\right) \\
+\left(t^{j+1}-t^{j}\right) \frac{\left(z_{i+0.5}-z_{i-0.5}\right)}{C \rho} \frac{\left(J_{i}^{j+0.5}\right)^{2}}{\sigma_{i}^{j+0.5}}
\end{gathered}
$$

where $\sigma$ depends on $T$. The current density is defined by approximating the potential (1) on a staggered grid which yields

$$
J_{i}^{j+1}=\sigma_{i} \frac{\phi_{i+0.5}^{j+1}-\phi_{i-0.5}^{j+1}}{z_{i+0.5}-z_{i-0.5}}, \quad \frac{1}{z_{i+1}-z_{i}}\left(J_{i+1}^{j+1}-J_{i}^{j+1}\right)=0 .
$$

Since all of the equations are coupled, the entire system (4), (5) must be solved simultaneously. But the fact that they form a nonlinear system means that this is potentially very demanding in terms of computing times. Normally, it takes only two iterations to reach a consistent solution of a fully coupled set (4) and (5) under smooth operation conditions, whereas modeling of the temperature surge event could require as many as 10 or even 20 iterations.

It is a common computational practice to separate source terms from the diffusion. The so-called "fractional steps" or

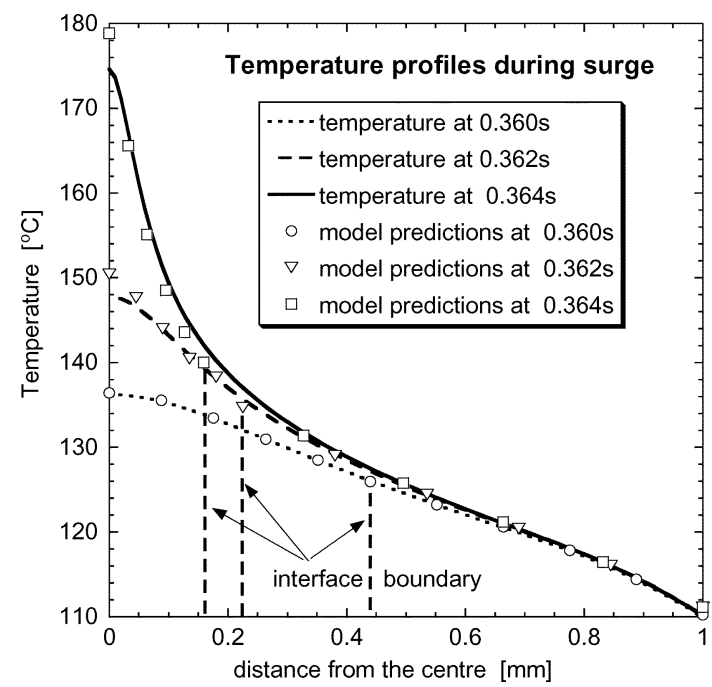

Fig. 3. Numerical predictions for temperature distribution in a thermistor at different moments of the surge stage by using the front-fixing technique. The nodes are automatically adjusted to the high-temperature region.

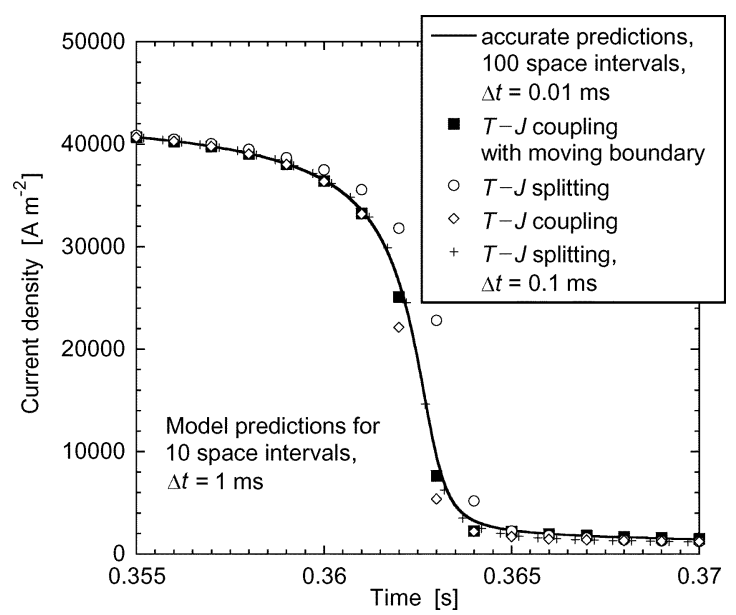

Fig. 4. Numerical predictions of current density during temperature surge in a thermistor.

"splitting methods" are widely used in computational fluid dynamic for the modeling of gas flow with chemical reactions [7], [8]. On the first substep, only diffusional fluxes in (4) contribute to temperature changes in a cell. Joule heat sources in (4) are added during the second substep, which is effectively the second-order Runge-Kutta integration. The splitting makes the computation iteration free, but requires small time steps for high accuracy to be achieved (Fig. 4). This is not surprising since large changes in the temperature profile at the surge stage (Fig. 3), due to Joule heat, are directly influenced by strong thermal diffusion.

\section{B. Front-Fixing Method}

Landau transformation utilizes new positional variables (one for each domain). In practical applications, the temperature rarely exceeds $T_{2}$. Only two domains with a boundary at $s=s_{1}$ need to be considered, constituting an additional simplification. In the plane case, an introduction of $u=z / s(t)$ fixes the extent 
of the low conductivity region $A$ to the domain $0 \leq u \leq 1$, while $v=(z-s) /\left(z_{\text {ext }}-s\right)$ fixes the extent of the other domain $B$ to $0 \leq v \leq 1$ [6]. Using the results from [9], the divergent form of (3) for $u$ and $v$, together with (1) for potential and interface equations at $s$, may be written as

$$
\begin{aligned}
\frac{\partial(C \rho s T)}{\partial t}= & \frac{d s}{\mathrm{dt}} \frac{\partial(C \rho u T)}{\partial u}+\frac{1}{s} \frac{\partial}{\partial u}\left(\kappa \frac{\partial T}{\partial u}\right) \\
& +s \sigma\left(\frac{\partial \phi}{\partial u}\right)^{2}, \\
\frac{\partial\left(C \rho\left(z_{\mathrm{ext}}-s\right) T\right)}{\partial t}= & \frac{d s}{\mathrm{dt}} \frac{\partial(C \rho(1-v) T)}{\partial v} \\
& +\frac{1}{z_{\mathrm{ext}}-s} \frac{\partial}{\partial v}\left(\kappa \frac{\partial T}{\partial v}\right) \\
& +\left(z_{\mathrm{ext}}-s\right) \sigma\left(\frac{\partial \phi}{\partial v}\right)^{2} \\
\frac{\partial}{\partial u}\left(\sigma \frac{\partial \phi}{\partial u}\right)= & 0, \quad u \in(0,1) ; \\
\frac{\partial}{\partial v}\left(\sigma \frac{\partial \phi}{\partial v}\right)= & 0, \quad v \in(0,1)
\end{aligned}
$$

with interface conditions for temperature and potential

$$
\begin{aligned}
& \left.\frac{1}{s}\left(\kappa \frac{\partial T}{\partial u}\right)\right|_{u=1}=\left.\frac{1}{z_{\mathrm{ext}}-s}\left(\kappa \frac{\partial T}{\partial v}\right)\right|_{v=0} \\
& \left.\frac{1}{s}\left(\sigma \frac{\partial \phi}{\partial u}\right)\right|_{u=1}=\left.\frac{1}{z_{\mathrm{ext}}-s}\left(\sigma \frac{\partial \phi}{\partial v}\right)\right|_{v=0} .
\end{aligned}
$$

It should be noted that the Landau transformation introduces a coordinate system in which all of the spatial boundaries are fixed to 0 or 1 . (Detailed illustration of the nodes' motion in real space is given in [9]). Under the transformation, the new computational domains remain the same with an additional advection term in (6) and (7) and nonlinear equations for the boundary motion (9) and (10). This allows treating the nodes close to the interface as being independent of the motion, which gives higher accuracy for the same number of nodes used. A divergent form of (6)-(8) ensures that there are no artificial energy sources [9]. The technique allows easy extension to 2-D geometry as shown in [6]. Its general application to 3-D models could be complicated, but often the transformation of only two coordinates is needed [6].

Equations (6)-(10) represent a typical Stefan problem with the so-called implicit moving boundary [6] and strongly nonlinear Joule heat source. The interface position is not strictly defined in this case and the displacement $s$ should be selected from physical considerations. The "effective" transformation temperature $T_{1}$ can be varied during the modeling in such a way as to achieve a better description of the transient process. The approach has already demonstrated a very high accuracy at sharp fronts, for example, in modeling an electric-field penetration into superconducting tapes [10].

Numerical scheme. In order to derive a finite volume scheme, space is discretized at $M+1$ points. The first $N+1$ points are defined by a fixed discretization of $u$, which corresponds to the extent of $A$. The points in $A$ are written as $u_{0}=0$, $u_{1}, \ldots, u_{N}=1$. The last $M-N+1$ points are in $B$ as given by a fixed discretization $v_{N}=0, v_{N+1}, \ldots, v_{M}=1$. The fi- nite volume discretization [8] of (6)-(10) is based on integration around the nodes and is fairly straightforward [9] (e.g., for the high-temperature phase $A$ )

$$
\begin{aligned}
&\left(T_{i}^{j+1} s^{j+1}-T_{i}^{j} s^{j}\right)\left(u_{i+0.5}-u_{i-0.5}\right) \\
&=\left(s^{j+1}-s^{j}\right)\left(\frac{T_{i}^{j+0.5}+T_{i+1}^{j+0.5}}{2} u_{i+0.5}\right. \\
&\left.-\frac{T_{i}^{j+0.5}+T_{i-1}^{j+0.5}}{2} u_{i-0.5}\right) \\
&+ \frac{t^{j+1}-t^{j}}{C \rho s^{j+0.5}}\left(\kappa_{i+0.5} \frac{T_{i+1}^{j+0.5}-T_{i}^{j+0.5}}{u_{i+1}-u_{i}}\right. \\
&\left.+\frac{t^{j+1}-t^{j}}{C \rho} s^{j+0.5}\left(u_{i+0.5}-u_{i-0.5}\right) \frac{\left(J_{i}^{j+0.5}-T_{i-1}^{j+0.5}\right.}{\sigma_{i}-u_{i-1}}\right)
\end{aligned}
$$

The interface equations are approximated in a consistent way to conserve energy [9] (e.g., for (9), $\left(h_{u}=1-u_{N-1}, h_{v}=v_{1}\right)$

$$
\begin{aligned}
& \left(T_{N}^{j+1} s^{j+1}-T_{N}^{j} s^{j}\right) \frac{h_{u}}{2} \\
& +\left(T_{N}^{j+1}\left(z_{\mathrm{ext}}-s^{j+1}\right)-T_{N}^{j}\left(z_{\mathrm{ext}}-s^{j}\right)\right) \frac{h_{v}}{2} \\
& =\left(s^{j+1}-s^{j}\right)\left(\frac{T_{N}^{j+0.5}+T_{N+1}^{j+0.5}}{2}\left(1-v_{0.5}\right)\right. \\
& \left.-\frac{T_{N-1}^{j+0.5}+T_{N}^{j+0.5}}{2} u_{N-0.5}\right) \\
& +\frac{t^{j+1}-t^{j}}{C \rho}\left(\kappa_{N+0.5} \frac{T_{N+1}^{j+0.5}-T_{N}^{j+0.5}}{h_{v}\left(z_{\mathrm{ext}}-s^{j+0.5}\right)}\right. \\
& \left.-\kappa_{N-0.5} \frac{T_{N}^{j+0.5}-T_{N-1}^{j+0.5}}{h_{u} s^{j+0.5}}\right) \\
& +\frac{t^{j+1}-t^{j}}{C \rho}\left(s^{j+0.5} \frac{h_{u}}{2}+\left(z_{\mathrm{ext}}-s^{j+0.5}\right) \frac{h_{v}}{2}\right) \\
& \times \frac{\left(J_{N}^{j+0.5}\right)^{2}}{\sigma_{N}^{j+0.5}}
\end{aligned}
$$

The interface position is defined implicitly by requiring that

$$
\begin{aligned}
T_{N}^{j+1}\left(\text { at } s^{j+1}\right)= & 0.6\left(T_{0}^{j+1}+T_{M}^{j+1}\right) \\
& \text { if } 0.6\left(T_{0}^{j+1}+T_{M}^{j+1}\right)<140^{\circ} \mathrm{C} \\
T_{N}^{j+1}\left(\text { at } s^{j+1}\right)= & 140{ }^{\circ} \mathrm{C}, \\
& \text { if } 0.6\left(T_{0}^{j+1}+T_{M}^{j+1}\right) \geq 140{ }^{\circ} \mathrm{C} .
\end{aligned}
$$

The particular choice in (13) ensures that the grid nodes are adjusted to the crucial location in the center of the thermistor.

The set of the simultaneous equations (11)-(13) involves the unknown future temperature field and potentials together with the future interface position. Since all of the equations are coupled, if the implicit scheme is to conserve energy, the entire system must be solved simultaneously. But the fact that they 


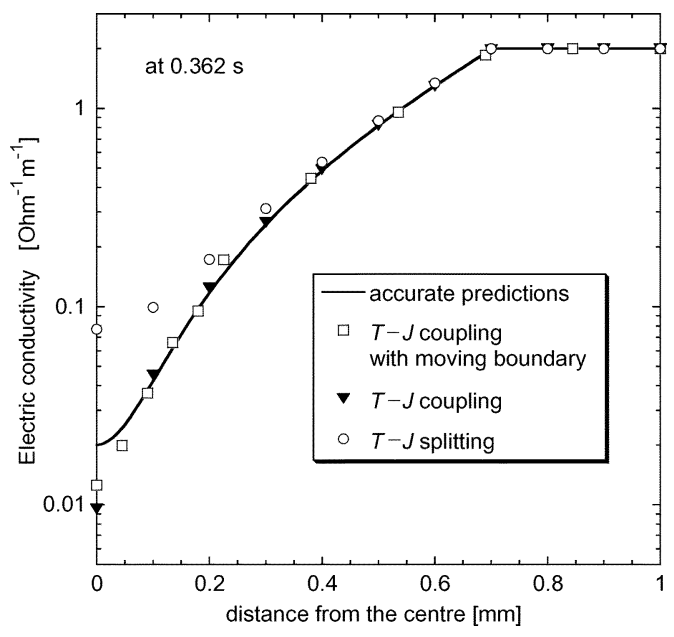

Fig. 5. Numerical predictions of electric conductivity in a thermistor in the middle of the surge stage. Coupled treatment provides high accuracy.

form a nonlinear system means that this is potentially very demanding in terms of computing times. It is interesting to note, however, that (6)-(8) as well as (9) and (10) are only weakly coupled; thus, if the future interface positions were known, the diffusion problem (6), (7), and the Poisson problem (8) would become quasilinear. With a known temperature profile, the potential could be found from (8) by using standard algorithms. Conversely, if future temperature and potentials were known, the future interface position could be calculated easily from (13). It is possible to derive an efficient algorithm based on decoupling the problem in this way. Normally, it only requires a few iterations to reach a consistent solution.

\section{TEMPERATURe Surge Modeling}

When a high current passes through the circuit, the temperature in the thermistor starts to rise. It takes a few tenths of a second to reach the low conductivity phase $\left(\sim 130^{\circ} \mathrm{C}\right)$ from the operational room temperature. At this stage, the surge takes place: the temperature increases by $\sim 50^{\circ} \mathrm{C}$ just in $5 \mathrm{~ms}$ (Fig. 3) and the current drops by two orders of magnitude (Fig. 4).

Looking at the predictions for electric conductivity (Fig. 5), it is obvious that conventional splitting methods fail to deliver sufficient accuracy during the surge at points with rapid temperature changes, as the method requires very small time steps to accomplish good accuracy, see Fig. 4. On the other hand, a fully coupled implicit method on a fixed grid provides good accuracy for temperature and electric conductivity at the grid nodes (Fig. 5). However, significant errors still exist for predicted current density (Fig. 4). This is related to the coarse discretization at crucial locations where the conductivity varies in an exponential manner. It should be noted here that the temperature variation is a secondary effect. The current density is very sensitive to a detailed description of high-temperature (low-conductivity) regions, and moving the coordinate system places the nodes at important positions. That is why the adjustment of nodes to the crucial location by moving the coordinate system yields excellent accuracy even for large time steps and a small number of discretization points.
TABLE I

COMPARISON OF COMPUTING TIMES

\begin{tabular}{|c|c|c|c|c|c|}
\hline Method & $\begin{array}{c}\text { Nu mber } \\
\text { of steps }\end{array}$ & $\begin{array}{c}\text { It erat ions } \\
\text { per step }\end{array}$ & $\begin{array}{c}T \\
\text { comput ians } \\
\text { per step }\end{array}$ & $\begin{array}{c}\phi \\
\text { computat ions } \\
\text { per step }\end{array}$ & $\begin{array}{c}\text { Overall } \\
\text { complexity }\end{array}$ \\
\hline Spl itt ing & 10 & 1 & 1 & 2 & 30 \\
\hline $\begin{array}{c}\text { Standard } \\
\text { Coupl ing }\end{array}$ & 2 & 7 & 1 & 1 & 28 \\
\hline $\begin{array}{c}\text { Moving } \\
\text { Coup ing }\end{array}$ & 1 & 11 & 1 & 1 & 22 \\
\hline
\end{tabular}

The splitting method utilizes a two-step Runge-Kutta integration and requires two solutions of Poisson equations at each step. To obtain the matching accuracy (Fig. 4), the standard coupling technique should use a smaller time step $\Delta t=0.5 \mathrm{~ms}$ compared with $\Delta t=1 \mathrm{~ms}$ for the front-fixing method.

Finally, as demonstrated in Table I, the conventional techniques exhibit similar performance, while the front-fixing reduces the computational effort by about a quarter.

\section{CONCLUSION}

The following conclusions can be drawn from this work:

1) There are several advantages in using a front-fixing method for modeling nonlinear diffusion processes in various electromagnetic phenomena, in particular, high accuracy can be obtained with a small number of grid points and large time increments.

2) Standard numerical methods for advection problems with diffusion can be utilized.

3) Bulk diffusion and complex interface conditions in the front-fixing method are usually weakly coupled. Efficient algorithms based on decoupling of the problem can be implemented.

4) High accuracy can be achieved on a coarse irregular mesh since the interface is automatically adjusted to crucial regions in new coordinates.

\section{REFERENCES}

[1] E. D. Macklen, Thermistors. Ayr, U.K.: Electrochemical Publications Ltd., 1979.

[2] A. C. Fowler, I. Frigaard, and S. D. Howison, "Temperature surges in current-limiting circuit devices," SIAM J. Appl. Math., vol. 52, no. 4, pp. 998-1011, 1992.

[3] X. F. Chen and A. Friedman, "The thermistor problem for conductivity which vanishes at large temperature," Quart. Appl. Math., vol. 51, no. 1 , pp. $101-115,1993$.

[4] A. S. Wood and S. Kutluay, "A heat balance integral model of the thermistor," Int. J. Heat Mass Transfer, vol. 38, no. 10, pp. 1831-1840, 1995.

[5] S. Kutluay and A. S. Wood, "Numerical solutions of the thermistor problem with a ramp electrical conductivity," Appl. Math. Comput., vol. 148, pp. 145-162, 2004.

[6] J. Crank, Free and Moving Boundary Problems. Oxford, U.K.: Clarendon, 1984

[7] J. C. Tannehill, D. A. Anderson, and R. H. Pletcher, Computational Fluid Mechanics and Heat Transfer, 2nd ed. Washington, DC: Taylor \& Francis, 1997.

[8] R. J. LeVeque, Finite Volume Methods for Hyperbolic Problems. Cambridge, U.K.: Cambridge University Press, 2002.

[9] T. C. Illingworth and I. O. Golosnoy, "Numerical solutions of diffusion-controlled moving boundary problems which conserve solute," $J$. Comput. Phys., vol. 209, no. 1, pp. 207-225, 2005.

[10] I. O. Golosnoy and J. K. Sykulski, "Evaluation of the front-fixing method capabilities for numerical modelling of field diffusion in high-temperature superconducting tapes," Inst. Eng. Technol. Sci., Meas. Technol., vol. 2, no. 6, pp. 418-426, 2008. 